\title{
A look at vacancies in dental practice
}

\author{
Vacant posts reported in dental practice: implications for human resource planning

\section{J. T. Newton and D. E. Gibbons. Br Dent J 2002; 192: 37-39}

\section{Objectives}

To describe the proportion of dental practitioners who report vacancies within their dental practice for the following: dental surgeons, dental hygienists, dental nurses and dental receptionists. To determine the proportion of dental practitioners working in general dental practice who would consider employing a dental therapist.

\section{Method}

Analysis of data from the British Dental Association Omnibus Survey 2000. The sample comprised 992 qualified dentists in all fields of practice.

\section{Findings}

The most commonly reported vacancies were for dental surgeons (18\% of practitioners reported vacancies), dental nurses (17\%) and dental hygienists (12\%). Approximately 46\% of general dental practitioners reported that they would employ a dental therapist if legislation permitted. The main reason for not employing a dental therapist was insufficient space in the dental practice.

\section{Conclusions}

A large number of vacancies exist for dental surgeons, dental nurses and dental hygienists in the United Kingdom. Around half of dental practices express an interest in employing dental therapists should legislation permit.

\section{IN BRIEF}

- Predicting the numbers of dental personnel required to provide dental services within the United Kingdom is an important but difficult task. The existence of vacant positions is a possible indication of a shortfall in these numbers.

- Approximately $18 \%$ of dental practioners nationally report vacant posts for dental practitioners within their practice of other place of work, $17 \%$ reported vacant posts for dental nurses and $12 \%$ reported vacant posts for dental hygienists.

- The employment of professionals complementary to dentistry is one alternative to filling a vacant post for a dental practitioner. Around half of general practices express an interest in employing dental therapists should legislation be introducted to permit this.

\section{COMMENT}

Planning workforce is an extremely difficult task to undertake.

There is no guaranteed way of obtaining the right answer. There are several ways in which the task can be approached. For example, population characteristics and the levels of disease, present and predicted, can be used to model likely workforce requirements. This method attempts to build workforce around the need for care. Others try to work around normal values by looking at ratios of dentists to population, assuming that some particular ratio is the desirable one. Still others try to look at the demand for dental care in the future and then adjust supply to meet that demand.

This study describes the requirement for workforce as measured by reported vacancies now. It is always very difficult to know how valid such a method is. Are there quirks in the system that mean there are a large (or small) number of vacancies at this particular time? Bias cannot be ruled out from a survey such as this and in particular a non-response bias. It may be that those who did not reply did not have vacancies. The risk of this is minimised in this study as the questions concerning vacancies were included as part of a wider survey.

What this survey achieves is a one-point in time snapshot of the need for additional dental workforce. As such it shows that there are a considerable number of vacancies for dentists and dental nurses in salaried services. Interestingly, there is a greater need for dentists in the north and dental nurses in the south.

There will always be vacancies as staff move jobs or retire or change direction. The problem is in identifying long term vacancies. Although it is not possible to do so from this study, the differences between areas and categories give some indication as to where there are problems. Certainly work from the West Midlands and anecdotal reports in Wales would support these findings that there are problems with recruitment.

Three UK countries are presently considering workforce issues. Scotland has published an interim report [Scottish Executive, 2000 \#4], Wales has a dental subgroup of a wider workforce review and England is in the middle of its review. This study is very useful in giving a national view of part of the problem concerning workforce.

Elizabeth Treasure

Professor of Dental Public Health

University of Wales College of Medicine 